\title{
Téoros
}

Revue de recherche en tourisme

\section{Économusées et tourisme}

\section{Hélène Deslauriers}

Volume 15, numéro 2, été 1996

Patrimoine industriel

URI : https://id.erudit.org/iderudit/1075030ar

DOI : https://doi.org/10.7202/1075030ar

Aller au sommaire du numéro

Éditeur(s)

Université du Québec à Montréal

ISSN

0712-8657 (imprimé)

1923-2705 (numérique)

Découvrir la revue

Citer ce document

Deslauriers, H. (1996). Économusées et tourisme. Téoros, 15(2), 51-54.

https://doi.org/10.7202/1075030ar d'utilisation que vous pouvez consulter en ligne.

https://apropos.erudit.org/fr/usagers/politique-dutilisation/ 


\section{ÉCONOMUSÉES ET TOURISME}

Hélène Deslauriers

Directrice générale

Fondation des économusées du Québec

Notre patrimoine, qu'il soit vivant ou architectural, ethnologique ou archéologique, industriel ou intangible, devrait, selon toute logique, offrir de nonbreuses occasions de développement touristique. Et pourtant, des nanifestations ou des sites se rapportant à notre patrimoine n'arrivent pas à développer l'intérêt attendu. Les économusées s'inscrivent dans une démarche de préservation et de mise en valeur du patrimoine en permettant aux visiteurs de comprendre et d'apprécier les savoir-faire traditionnels. Les économusées suscitent actuellement beaucoup d'intêrêt de la part des différents intervenants et des médias. Les demandes d'information sont nombreuses et la couverture médiatique abondante. S"agit-il d'une attention particulière dont fait l"objet tout nouveau produit touristique? Est-ce plutôt la réponse favorable à une formule différente des autres attractions à caractère patrimonial?

Nous tenteron, dans cet anticle, de mieux cerner le concept d'économusée, ses objectifs et ses créneaux, d'expliquer en quoi les écononusée se démarquent des autres attractions touristiques et de comprendre conunent ils participent à la valorisation du parrinoine artisanal et industriel.

\section{LES CONDITIONS DE SUCCES}

Au départ les objectifs de l'économuséologie étaient de:

- promouvoir un patrimoine qui sgagne sa view;

- conserver le meilleur de la tradition;

- développer le tourisme culturel et scientifique.'

Pour y arriver, six différentes fonctions doivent $s^{\prime} y$ retrouver:

- L'accueil des visiteurs

- Les ateliers de production

- L'interprétation du passé

- L'interprétation de la production actuelle
- Le centre de documentation

- La boutique ou galeric

La Papeterie Saint-Gilles, depuis près de dix ans, et huit autres entreprises ont servi de lieu d'expérimentation. Parmi cellesci, on compte quelques entreprises privées où le producteur ou l'artisan est présent. Dans d'autres cas, il s'agit plutôt d'un site ou certaines parties d'une production traditionnelle sont présentées au public sous forme de démonstrations et où l'aspect muséal prend plus d'importance.

Il semblait évident, au départ, que la formule permettrait à certains musées de rentabiliser leurs opérations. Sans nier cette possibilité, l"expérience nous oblige à nuancer cet aspect. On constate en effet, qu'à partir de l'évaluation des résultats obtenus dans les neuf entreprises, réalisée sur la base des commentaires des visiteurs, de sondages et de l'appréciation même des propriétaires d'entreprises économuséales, la difficulté d'introduire dans un musée une production authentique, complete et répondant au besoin du marché.

Mềme si les neuf économusées connaissent presque tous une amélioration de leur situation depuis leur entrée dans le réseau, nous pouvons affirmer maintenant que certaines conditions de base sont essentielles au succès du projet. Ainsi, les entreprises qui obtiennent de meilleurs résultats sont celles où l'on retrouve un artisan ou un producteur dans son milieu quotidien, qui fait la démonstration de la fabrication de son produit, explique ses techniques et qui répond avec compétence aux questions des gens. Le fait que les visiteurs puissent acheter des produits fabriqués sur place, sous leurs yeux, ajoute à l'intérêt de la visite.

D'autres moyens d'interprétation complettent la visite et fournissent des informations supplémentaires. La qualité et l'originalité de ces moyens comptent pour beaucoup. Toutefois, à eux seuls, ils ne procurent pas aux visiteurs l'élément distinctif qui leur fait apprécier l'ecconomusée comme equipement culturel différent. C'est pourquoi le choix des économusées se fait désormais sur la base d'une définition plus précise soit une entreprise privée qui : 


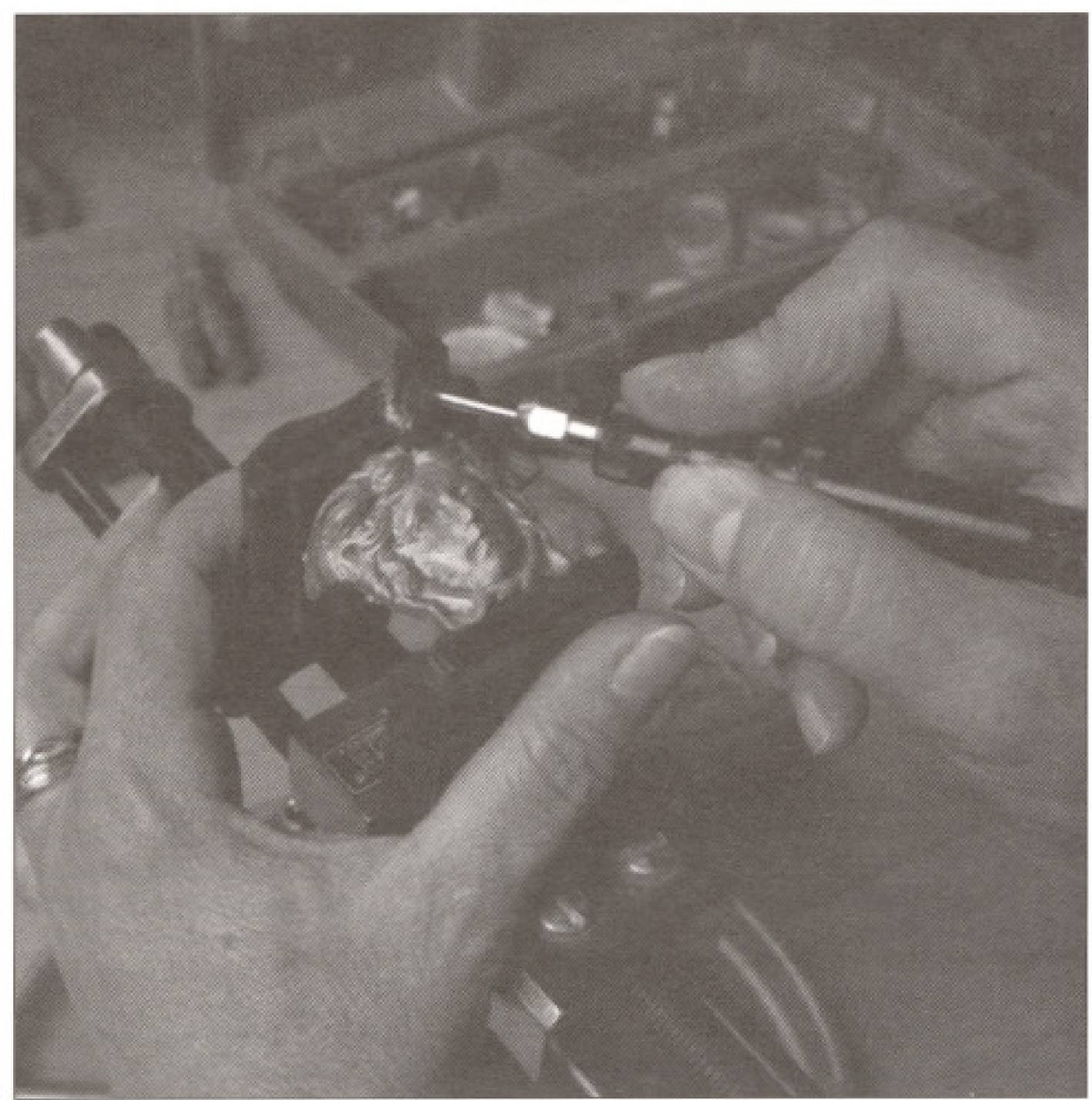

Produit de la micro-fonderie, écononusée du bronże, Invernéss.

- utilise pour sa production une technique ou un savoir-faire traditionnel;

- ouvre ses portes au public afin de meltre en valeur ces savoir-faire et ces artisans de chez-nous;

- est dotée d'un lieu d'animation et d'interprétation de la production.

Il s'agit donc sans contredit d'un équipement culturel, plus près cependant de la visite industrielle puisqu'il permet au public de s'introduire dans une entreprise en production et de pouvoir assister aux étapes quotidiennes de travail.

Parmi les critères de sélection utilisés actuellement pour le choix des économusces, on retrouve prioritairement les élếments suivants :

- l'entreprise doit être rentable;

- elle doit exister depuis plus d'un an;

- elle doit générer un chiffre d'affaires de plus de 50000 \$:

- elle doit être gérée par un ou des artisans.
S ajoutent a cela des criteres concernant l'aspect traditionnel de la production, la localisation, le caractere des lieux et la capncilé des artisans â accueillir des visiteurs. Celte dófinition el ces criteres ne remettent aucunement en question les objectifs de départ, ils ne font que préciser la manière et les moyens de les atteindre.

\section{L'ÉCONOMUSÉE COMME PRODUIT TOURISTIQUE}

Avant d'être des économusêes, les sites el les premières entreprises ouvraient déja leur porte aux visiteurs. Les informations fournies visaient tếnéralement à répondre aux questions les plus récurrentes. Les aspects historiques de la production et l'evolution des techniques ou de I'entreprise prenaient une place importante. On pouvait cependant constater que la majorité des économusées se prêtaient à l'interprétation et à la vulgarisation de sujets scientifiques ou technologiques qui fascinaient le public : la transformation du suc des fleurs en miel, le conlage du bronze, les propriêtés particulières du verre.

Cette constatation déboucha sur la réalisation de projets pilotes dans le domaine de la culture scientifique avec le ministère de l'Industrie, du Commerce, de la Science et de la Technologie. L'expérience nous permet maintenant d $d^{\dagger}$ laborer les contenus offerts aux visiteurs sur la base d'un modèle où les aspects historiques et scientifiques se complètent, et ce sous cinq grands thèmes :

- la matière première, ses propriétés, ses avantages, son approvisionnement, etc.;

* l'artisan et son métier, les cycles de production, les habiletés nécessaires, l"évolution du métier dans le temps, son adaptation au monde contemporain;

* les techniques et les outils, les façons de faire traditionnelles et contemporaines, la machinerie, les traces du métier;

- la ligne de temps, l'historique de cetie production ou d"un aspect en particulier du métier;

- les phénomènes scientifiques et technologiques, parfois spectaculaires (la fermentation du cidre), mais souvent intangibles (I'acidité de certains bois, élément répulsif pour les termites).

Une fois le contenu ả offrir défini, il s'agit d'identifier les moyens les plus efficaces pour le transmettre.

\section{L'APPROCHE EN INTERPRÉTATION}

Nous I'avons déjả constaté : I'atelier ou le lieu de production et l'artisan producteur constituent les meilleurs outils d'interprétation. Qui n'a pas déjà eu envie de voir fonctionner un moulin à vent ou d'assister à la créstion d'un vitrail ou à la fabrication des menus accessoires d'un bateau miniature ?

Les visiteurs recherchent ces occasions de suivre la fabriquation des produits qu'ils pournont acheter ou qu'ils consomment à l'occasion. Plus encore, ils apprecient le fait de puiser les informations aupres d'un authentigue producteur, qui 
exerce ce métier et qui souvent l'a vu s'exercer pendant des années auparavant. Ils aiment tout particulièrement avoir l'impression d"entrer dans la vie des gens et de vivre auprès d'eux une expérience unique.

L'atelier ou le lieu de production, mais surtout l'artisan livrant son savoir, deviennent donc le coeur de l'économusée avec les contraintes que supposent le cycle de production, la disponibilité des gens, les heures d'ouverture, etc.

Pour palier ces inconvénients d'autres moyens s'ajoutent: panneaux d'information, bornes interactives, vitrines d'objets anciens ou contemporains. Pour les plus exigeants, un petit centre de documentations permet de puiser davantage de connaissances dans des ouvrages spécialisés ou des revues. Ces moyens doivent cependant être choisis minutieusement en fonction de la nature même du metier et de la personnalité des artisans qui participent d'ailleurs activement à l'élaboration du concept.

L'artisan producteur doit lui-mềme aimer et assumer ce contact avec le public et tenter, le plus possible, d'être la source principale de transmission des informations. Lorqu'en plus, d'autres membres de la famille ou d'autres associés participent ă l'accueil des visiteurs, la visite s'enrichit. Aux dires de certains propriétaires, il existe d'ailleurs un lien direct entre la durée de la visite, la qualité du contact avec l'artisan et le montant dépensé en achat de produits.

\section{LE CIRCUIT TOURISTIQUE}

Le succès du réseau des bconomusées ou du moins l'intêrêt qu'on lui témoigne réside, sans aucun doute aussi, dans l"a= vantage de proposer un circuit qui couvrira sous peu le Québec. Les agences touristiques et les promoteurs apprécient le fait d'offrir à leur client plusieurs destinations au cours d'un mêne pếriple d'autant plus s'ils peuvent compter sur un réseau homogène dans la qualité de l'accueil et la richesse des experiences vécues.

Pour cette raison, le réseau se développe par boucles et concentrations. La presence de deux ou plusieurs economusées

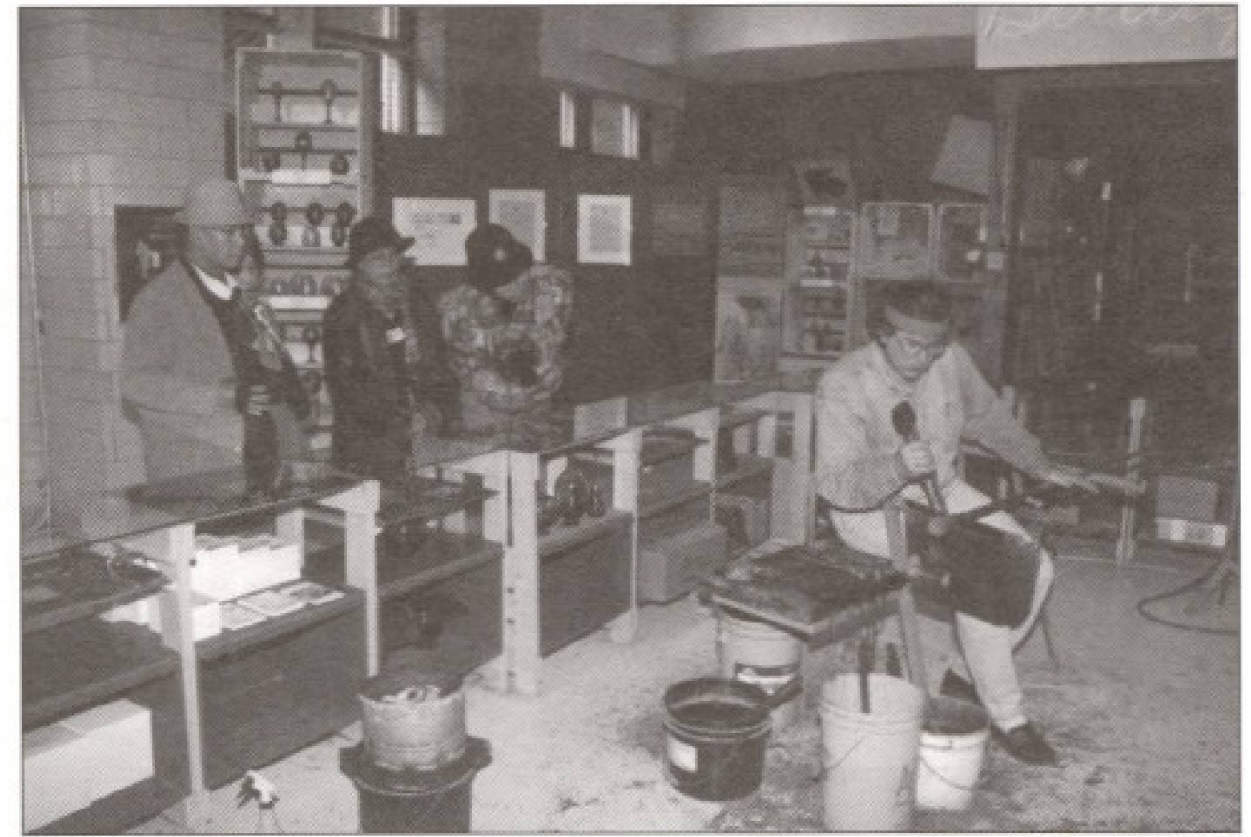

Jean Vallières, maître verricr, à l'économuste du verre, à Québec.

dans une région crểe l'auraction nécessaire pour inciter le visiteur à s'y rendre. Ainsi, la majorité des économusées se retrouvent à Québec, dans Charlevoix et sur la route qui relient ces deux pôles.

Le choix des six demiers projets s'est fait dans le but de consolider les premières implantations : une deuxième entreprise à Québec, l'économusée du vitrail, les économusể de l'enseigne sur bois à Lévis et des bateaux miniatures à SaintJean-Port-Joli entre ceux de Montmagny (Manoir de l'accordéon) et de SaintAndre-de-Kamouraska (la Maison de la prune), et enfin l'économusếe du cuir à Victoriaville pour enrichir le réseau autour de l'économusée du bronze d'Inverness. Seul le choix de la Cidrerie à Mont-Saint-Hilaire permet d'ouvrir ce qui deviendra le circuit vers Montreal. Les prochaines sélections d'entreprises répondront aux mêmes objectifs de stabilisation des circuits existants et d"ouverture de nouvelles routes pour toucher les régions de la Rive-Nord, des Laurentides, de l'Estrie, de l'Outaouais, elc.

\section{LA NOTORIÉTÉ D'UN RÉSEAU}

L'intérêt pour les entreprises d'adhérer à ce réseau et d'en accepter les principes et les modes de fonctionnement réside dans l'image particulière et la notoriété que leur confere le fail d'avoir été choisis dans ce groupe comme exemple unique dans leur domaine. Plusieurs propriétaires nous ont confié leur besoin de se démarquer ainsi de leurs concurrents.

Pour cette raison, les membres du réseau se préoccupent du choix des entreprises. Conscients que la qualité de chacun des économusées influence la perception du visiteur et que le succes ou l'insucces des autres rejaillissent sur eux, ils souhaitent être associés à des projets performants et de qualité.

Il ne s'agit donc pas uniquement de sélectionner les projers, encore faut-il s"assurer que toutes les conditions de succès s'y retrouvent et que les entreprises puissent évoluer dans les meilleures conditions afin de maintenir leur qualité et se renouveler. Dans ce sens, la Fondation joue un rôle important dans la mise en marché des économusées, et travaille constamment à favoriser les projets de jumelage ou le développement de nouveaux produits et à les mettre en contact avec les informations et l'expertise qu'ils requièrent.

\section{LES CLIENTÈLES}

Pour l'instant, aucune étude systématique des clientèles n'a êté faite dans le réseau. Certains économusées connaissent bien leurs clients et les questionnent régulière- 


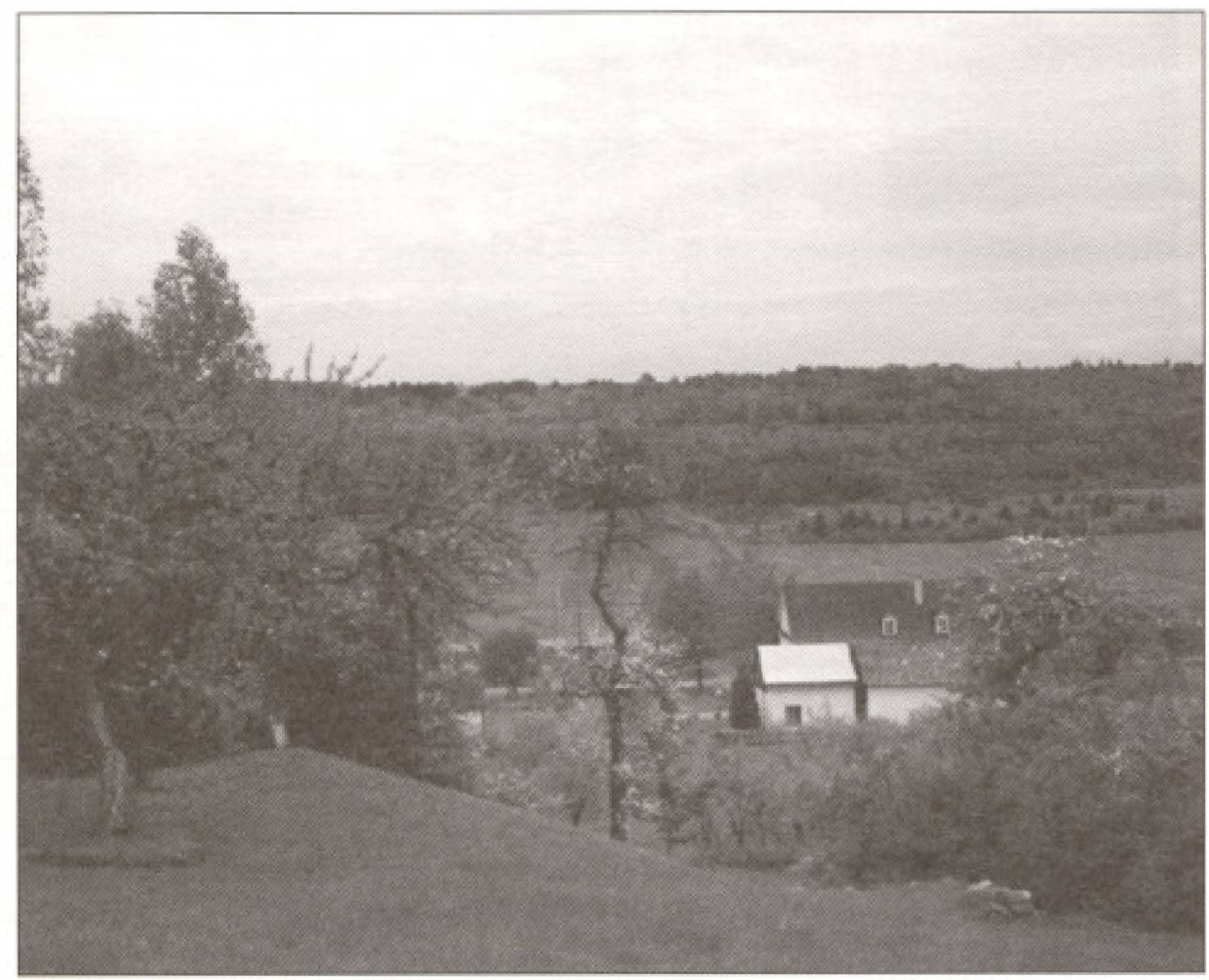

Les vergers et la Maison de la prune, économusée de la prune, Saint-André de Kanouraska.

ment alors que la plupart enregistre precisément le nombre de visiteurs. Avec la venue des treize nouveaux projets (sélections de mai et octobre 1996), l'intérêt d'identifier les types de visiteurs, leur profil, leurs besoins se fera davantage sentir. Dans certains cas, les études de marché viseront aussi à prévoir la réponse des clientèles en fonction d'un projet particulier.

\section{LE DÉFI DU RÉSEAU}

Le concept de l'économusée connaît en ce moment un succès certain. Les réactions et les commentaires sont généralement positifs et enthousiastes. Ce produit touristique comporte bien sûr certains inconvénients inhếrents à la formule même :

- la production peut être différente selon les saisons (un verger ne produit pas l'hiver, les abeilles ne sortent que durant les mois plus doux);

- les visites peuvent coïncider avec des périodes moins spectaculaires de la production;

- le producteur ou l'artisan doit parfois s'absenter et priver le visiteur de ce contact;
- dans certains cas, l'ouverture au public se résume à quelques mois de l'année.

Bien qu'il faille travailler à réduire ces contraintes au minimum, le visiteur doit pour sa part reconnaître qu'elles participent souvent à préserver l'authenticité de l'expérience. En retour, les économusées offrent au public des équipements culturels vivants, dynamiques et rentables (la seule aide linancière leur étant fournie au moment de leur transformation en économusée). Parce qu'ils sont supportếs par une entreprise commerciale saine et compétitrice, les économusées évoluent au rythme de cette entreprise, se renouvellent et innovent tout en gardant une place importante à la tradition.

À l'heure où tout se robotise et où l'ordinateur remplace de plus en plus le geste humain, les gens veulent savoir comment se font les choses et comparer ces méthodes avec les techniques d'autrefois. Que ce soit pour le pot de miel, le sac en cuir ou la goślette, ils apprécient l'ingéniosité humaine derrière les produits qu'ils consomment ou utilisent. Mais ils exigent davantage : ils veulent que ces informations leur soient transmises de façon authentique sans artifices (ou très peu), le plus simplement possible par des gens compétents, des producteurs et des artisans qui connaissent leur métier et peuvent répondre à leurs nombreuses questions.

Le défi du réseau des économusées réside done dans le choix minutieux des artisans et des entreprises et dans leur mise en valeur et leur transformation en lieux accueillants, sans toutefois mettre en péril l'authenticité des lieux et de leurs propriétaires.

Ces dernières constatations nous portent à croire qu'il en est de même pour les autres éléments et manifestations de notre patrimoine industriel ou technologique souvent négligé et difficile à vendre. S'il constitue une source d'intérêt importante dans le public, la mise en valeur de ce patrimoine doit cependant se faire de façon simple, en présentant aux visiteurs des informations pertinentes de façon vivante et dynamique.

\section{NOTE BIBLIOGRAPHIQUE}

1 Simard, Cyril. Économusée, Eoonomuseum, Economusco, Fondation des economusées du Québec, Québec, 1992, 21 p. 